\title{
Qualitätsmanagement in der Versorgung
}

\author{
Birgit Janssen, Ralph Menke, Wolfgang Gaebel
}

Klinik und Poliklinik für Psychiatrie und Psychotherapie der Heinrich-Heine-Universität

Düsseldorf, Rheinische Kliniken Düsseldorf

psychoneuro 2004; 30 (11): 620-623

Die Steigerung von Qualität und Effizienz in der gesundheitlichen Versorgung gewinnt in der politischen Diskussion zunehmend an Bedeutung. Auch im Bereich der neuro-psychiatrischen Erkrankungen mehren sich seit einigen Jahren Bestrebungen, Optimierungspotentiale zu identifizieren und durch stärkere ärztliche Leitlinienorientierung im Behandlungsprozess zu erschließen. Die schizophrenen Störungen erscheinen aufgrund ihrer medizinischen, gesundheitspolitischen und ökonomischen Bedeutung in besonderer Weise geeignet zur exemplarischen Entwicklung und Erprobung von Verfahren des Qualitätsmanagements in der Psychiatrie. Bei der Neuentwicklung von QM-Methoden und Instrumenten bzw. bei ihrer Übertragung aus den somatischen Fächern sollten jedoch psychiatriespezifische Besonderheiten wie die schwierige Operationalisierung des Behandlungsergebnisses beachtet werden. Der Beitrag geht zunächst kursorisch auf den gegenwärtigen Stand des Qualitätsmanagements in der Psychiatrie ein und stellt dann eine Reihe von Projekten zur Qualitätsoptimierung in der Schizophreniebehandlung unter dem Dach des Kompetenznetzes Schizophrenie vor.

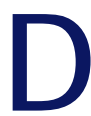
ie gegenwärtige gesundheitspolitische Diskussion wird nachhaltig durch Forderungen nach mehr Qualität und Effizienz im Gesundheitswesen bestimmt. Auch die psychiatrische Versorgung stellt sich mit zunehmender Aufmerksamkeit Fragen nach Behandlungsvariationen, Leitlinienkonformität in der Behandlung und der Erschließung möglicher Optimierungsspielräume. Verglichen mit anderen medizinischen Teildisziplinen haben systematische Bemühungen um Qualitätssicherung in der Psychiatrie in Deutschland eine relativ junge Geschichte. Seit gut zehn Jahren sind jedoch auch in der psychiatrischen Versorgung zunehmend Bestrebungen zu erkennen, den Anschluss an den erreichten Stand in anderen medizinischen Dis- ziplinen zu sichern. Qualitätssicherung und Qualitätsmanagement in der Psychiatrie können dabei prinzipiell auf alle Aspekte therapeutischen Entscheidens und Handelns in den Bereichen Diagnostik, Therapie, Rehabilitation und Nachsorge ausgedehnt werden, unter Berücksichtigung der jeweiligen institutionellen und versorgungspolitischen Rahmenbedingungen.

„Quality of care is the degree to which health services for individuals and populations increase the likelihood of desired outcomes and are consistent with current professional knowledge" lautet eine bekannte Definition des amerikanischen Institute of Medicine aus dem Jahre 1990 (IOM). Diese Beschreibung findet ihre Entsprechung in den Regelungen des Sozialgesetzbuch V, wonach eine bedarfsgerechte, dem Stand der medizinischen Erkenntnisse entsprechende Versorgung der Versicherten gefordert ist. Von Leistungserbringern und Krankenkassen wird jedoch weiterhin erwartet, hierbei den Rahmen des Notwendigen nicht $\mathrm{zu}$ überschreiten und eine wirtschaftliche Erbringung der Behandlungsleistungen zu sichern (vgl. § 70 SGBV). Die Gesundheitsversorgung soll idealerweise einen Ausgleich zwischen Qualität, Humanität und Wirtschaftlichkeit erreichen. Der Gesetzgeber hat zudem eine Verpflichtung zur Sicherung und Weiterentwicklung der Versorgungsqualität festgeschrieben (vgl. 135a SGBV). Krankenhäuser der Regelversorgung sind nach dem Sozialgesetzbuch aufgefordert, ein klinikinternes Qualitätsmanagement einzurichten und sich an einrichtungsübergreifenden Maßnahmen der Qualitätssicherung - beispielsweise in Form von Qualitätsvergleichen zu beteiligen, womit insbesondere eine Optimierung der Ergebnisqualität angezielt wird. Niedergelassene Ärzte, Zahnärzte und Psychotherapeuten sind ebenfalls zu praxisübergreifender Qualitätssicherung und seit dem Gesundheitsmodernisierungsgesetz (GMG) - zur Einrichtung eines internen Qualitätsmanagements verpflichtet. Unabhängig von der aktuellen Diskussion haben 
Maßnahmen zur Sicherung der diagnostischen und therapeutischen Qualität innerhalb der ärztlichen Profession natürlich bereits eine lange Tradition. Hierzu zählen bspw. die Krankenakte als Behandlungsdokumentation, Visite, Konsil oder Supervision, die Teilnahme an Fortbildungen oder Ring-Tests. Diese vielfach gut eingeführten Maßnahmen dienen zunächst der Sicherung der erreichten Behandlungsqualität, die einzelnen Ansätze blieben jedoch unverbunden und - gemessen an der Forderung nach einem umfassenden Qualitätsmanagement relativ unstrukturiert.

Bisherige Versuche, im Bereich der Psychiatrie Instrumente und Methoden des Qualitätsmanagements zu entwickeln oder anzuwenden, griffen vielfach auf entsprechende Erfahrungen aus den somatischen Fächern oder gar direkt aus dem Industrie- bzw. Dienstleistungssektor zurück. Die medizinische Versorgung neuro-psychiatrischer Erkrankungen weist jedoch auch gegenüber anderen Krankheitsbildern - Besonderheiten auf, denen Rechnung zu tragen ist. Dazu gehören bspw. in der Regel stark individualisierte Krankheitsverläufe, eine Kombination von Medikation und „sprechender“ Medizin, erhebliche Belastungen für Patient und Angehörige sowie eine schwierige Operationalisierung der Erfolgsmessung.

Als verdienstvoll muss daher zunächst die Entwicklung einer Psychiatrischen Basisdokumentation (BADO) durch Cording und Kollegen angesehen werden. Wenn sich die BADO dennoch nicht im erhofften Maße hat durchsetzen können, werden als Ursachen u.a. abnehmende Datenqualität unter Routinebedingungen geltend gemacht sowie die Beschränkung auf krankheitsübergreifende Skalen zur Ergebnisdokumentation mit ungenügendem spezifischen Aussagewert (16). Ein Ausweichen auf routinemäßig generierte administrative Daten verbietet sich, da erhebliche technische und methodische Probleme zu erwarten sind (16). Das Problem einer aussagefähigen, auch unter Alltagsbedingungen einsetzbaren, EDV-fähigen psychiatriespezifischen Qualitätsdokumentation kann demnach bislang noch als offen angesehen werden.

Eine für die Bundesrepublik relativ neue Entwicklung stellt zudem die Diskussion um die Entwicklung und Implementierung von Behandlungsleitlinien im Interesse einer stärkeren Evidenzbasierung des ärztlichen Handelns dar. Im angloamerikanischen Raum hat sich die Entwicklung von Leitlinien zu Diagnostik und Therapie bereits seit längerem etablieren können, in Europa kommt vor allem Großbritannien und den Niederlanden eine führende Rolle in der Leitliniendiskussion zu.

In der Bundesrepublik erhielt der Themenbereich Leitlinien jedoch breiten Auftrieb durch die im GKVGesundheitsreformgesetz 2000 angestrebte Verankerung des Prinzips der evidence based medicine (EBM) im Bereich ambulanter und stationärer Leistungen ( $§ 136-\S 137$ e SGBV). Definitorisch müssen Leitlinien zunächst abgegrenzt werden von Empfehlungen, wie sie bspw. in medizinischen Lehrbüchern oder Fachzeitschriften publiziert werden, sowie von Richtlinien als Handlungsoder Unterlassungsregelungen rechtlich hierzu ermächtigter Institutionen, deren Nichtbeachtung definierte Sanktionen nach sich zieht. Leitlinien sollten demgegenüber als Orientierungshilfen im Sinne von Entscheidungskorridoren verstanden werden, die selbstverständlich eine Abweichung in begründeten Fällen einschließen und nicht zwangsläufig auf eine Begrenzung der ärztlichen Autonomie hinauslaufen $(5,14)$. Wissenschaftliche Fachgesellschaften und ärztliche Selbstverwaltung haben zwischenzeitlich eine Reihe gemeinsamer Qualitätsanforderungen für die Leitlinienentwicklung formuliert (1).

Anfänglich in der Bundesrepublik insbesondere auf Initiative einzelner Fachgesellschaften vorgelegte Leitlinien sind daher überwiegend noch der Stufe I (Erarbeitung und informelle Autorisierung durch Expertengruppe) bzw. Stufe II (Formale Konsensusfindung einschließlich Evidenzbewertung) zuzuordnen, während künftige Leitlinien sich im Interesse höchstmöglicher Evidenzbasierung an der Stufe III (Leitlinie mit allen Elementen systematischer Erstellung) orientieren sollten. Für den Bereich der neuro-psychiatrischen Erkrankungen sei hier stellvertretend auf die durch die Deutsche Gesellschaft für Psychiatrie, Psychotherapie und Nervenheilkunde (DGPPN) herausgegebene Reihe von Praxisleitlinien hingewiesen, deren erste sich mit der Schizophrenie befasste (3). Eine Überarbeitung steht kurz vor dem Abschluss, sodass in Kürze eine evidenzbasierte Stufe-III-Leitlinie für die Behandlung schizophrener Störungen zur Verfügung steht.

In Anlehnung an den eingangs zitierten $\S 70$ des Sozialgesetzbuch V erscheinen Leitlinien grundsätzlich geeignet, einen Beitrag zum Abbau von Über- oder Fehlversorgung und zur Mobilisierung von Qualitätsund Wirtschaftlichkeitsreserven zu leisten. Eine stärkere Orientierung der ärztlichen Entscheidung an evidenzbasierten Leitlinien in Diagnostik und Therapie lässt eine Optimierung des Behandlungsprozesses erwarten, verbunden mit verbesserten Behandlungsergebnissen und der Mobilisierung von Wirtschaftlichkeitsreserven (5). Versuche zur Implementierung von Leitlinien in die Versorgungslandschaft sind in der Vergangenheit jedoch wiederholt auf Widerstände innerhalb der ärztlichen Profession gestoßen, die unter anderem durch prinzipielle methodische Vorbehalte gegenüber der evidenzbasierten Medizin, das Gefühl einer Überflutung mit Leitlinien oder Angst vor einem Verlust ärztlicher Autonomie begründet waren (17). Dennoch konnte in Übersichtsarbeiten eine Reihe erfolgversprechender Strategien zur Überwindung von Implementationsbarrieren identifiziert werden $(8,9)$ :

- Gezieltes Aufsuchen von Leistungserbringern durch Peers oder andere akzeptierte Experten, qualitätsbezogenes Feedback

- Verwendung fallbezogener, leitlinienbasierter Erinnerungssysteme (insbesondere bei Nutzung EDV-unterstützter Lösungen) 
- Interaktive Elemente im Rahmen von Fortbildungsveranstaltungen bzw. eLearning

- Den nachhaltigsten Interventionserfolg zeigen aufwändige integrierte Vorgehensweisen unter Kombination der genannten Einzelelemente.

\section{Verbesserung der Schizo- phreniebehandlung durch Leitlinienimplementierung}

Die schizophrenen Störungen sind von erheblicher gesundheitspolitischer Bedeutung. Trotz deutlich verbesserter Behandlungsmethoden und -erfolge in den letzten Jahrzehnten kommt es vielfach noch zu chronifiziertem Krankheitsverlauf, mit Einschränkungen der Berufs- oder Erwerbsfähigkeit. Behandlungs- und Folgekosten machen die Schizophrenie zur teuersten psychiatrischen Erkrankung überhaupt. Die Behandlung ist in der Regel mit wiederholten Wechseln zwischen ambulanter allgemein-/ fachärztlicher und stationärer Versorgung verbunden, die erhebliche Potentiale zur Verbesserung der ambulant/stationären Schnittstelle erwarten lassen. Es bestand daher die Absicht, unter dem Dach des Kompetenznetz Schizophrenie anhand der Tracerdiagnose schizophrene Störungen systematisch Instrumente und Methoden des internen und externen Qualitätsmanagements zu entwickeln und modellhaft zu evaluieren. Zudem sollte eine Bestandsaufnahme des gegenwärtigen Standes der ambulant/stationären Schizophreniebehandlung hinsichtlich Leitlinientreue und definierter Qualitätsindikatoren erfolgen. Hierbei konnte auf vorhandene Studien (4) sowie auf eigene Vorarbeiten zurückgegriffen werden. So standen zum einen die Praxisleitlinien der DGPPN zur Verfügung, zum anderen konnten im Rahmen einer Tracerstudie Schizophrenie (6, 11) Empfehlungen zum externen Qualitätsmanagement in der stationären Schizophreniebehandlung vorgelegt werden. Die vorliegenden Erfahrungen sollten daher zu einem umfassenden Modell internen und externen Qualitätsmanagements zusammengeführt werden, dass so- wohl in einem ambulanten nervenärztlichen Setting als auch in Klinikverbünden implementiert werden sollte. Die geplanten Interventionen umfassten eine qualitätsorientierte Behandlungsdokumentation, Zusammenführung der klinikbzw. praxisspezifischen Daten einschließlich anonymisierter Qualitätsrückmeldung (Benchmark) als Basis für Schwachstellen-Identifizierung und gezielte leitlinienorientierte Optimierung von Behandlungsprozessen entsprechend dem PDCA-Zyklus. Ziel der geplanten Intervention war eine Optimierung von Prozess- und Ergebnisqualität durch Besserung der Leitlinienorientierung in Diagnostik und Therapie, sowie einer Reduzierung von Qualitätsunterschieden in der Schizophreniebehandlung.

\section{Qualitätsmanagement in der Schizophreniebehandlung - vorliegende Ergebnisse}

Nach den bislang vorliegenden Ergebnisse ergeben sich Hinweise auf Varianzen hinsichtlich der Prozess- und Ergebnisqualität in der Schizophreniebehandlung. Weiterhin zeigt sich vielfach eine noch mangelnde Leitlinientreue in der ärztlichen Entscheidungsfindung.

Im Folgenden sollen vier aktuelle Projekte unter Federführung der Rheinischen Kliniken Düsseldorf kurz erläutert werden.

- Leitliniengestütztes Qualitätsmanagement in der stationären Akutbehandlung schizophrener Patienten: Das Projekt untersuchte Behandlungsergebnisse und Leitlinienkonformität in sieben psychiatrischen Fachkliniken in Nordrhein-Westfalen und Baden-Württemberg $(12,13,18)$. In einem Kontrollgruppendesign erhielten die Kliniken der Interventionsgruppe qualitätsbezogene Benchmarks als Grundlage für Qualitätszirkelarbeit

- Ein Ergebnistransfer aus dem stationären Projekt erfolgt aktuell im Rahmen eines BMGS-geförderten Projekts „Benchmarking in der psychiatrischen Akutbehandlung - Ein Modellprojekt im Klinikverbund“, an dem sich alle neuen psychiatri- schen Fachkliniken des Landschaftsverbandes Rheinland beteiligen

- Leitliniengestütztes Qualitätsmanagement in der Nervenarztpraxis: Im Rahmen dieses Projekts wurde ein EDV-gestütztes leitlinienbasiertes Decision-Support-System entwickelt und in einem Düsseldorfer nervenärztlichen Praxisnetz evaluiert. Als zweite Interventionsgruppe fungierten Praxennetze in Freiburg bzw. München. Das EDV-Modul umfasst neben einer qualitätsorientierten Dokumentation eine interaktive, fallbezogene Leitlinienvermittlung für den behandelnden Arzt einschließlich Entscheidungsunterstützung in Algorithmenform (10, 15). Im Rahmen der Intervention konnte ein positiver Effekt des DecisionSupport-Moduls hinsichtlich Leitlinientreue der Behandlungsprozesse und Ergebnisqualität gezeigt werden

- Das Schizophrenie-Modul wird gegenwärtig bereits in weiteren Praxennetzen eingesetzt sowie in einem Projekt zur Verbesserung der nachstationären Versorgung schizophrener Patienten, dass durch eine Gruppe von Krankenkassen gefördert wird.

Quality management in health care The improvement of quality and efficacy in health care becomes more and more important in the political discussion. Even in the area of neuropsychiatric diseases efforts can be seen during the lasts years to identify potentials for optimisation, and to open them up by increasing guideline adherence in physician's decisionmaking in the treatment process. Due to its importance in medicine, health policy and economy schizophrenic disorders seem to be particularly suitable for exemplary development and evaluation of quality management methods in psychiatry. However, new developments of QM-methods and instruments or transfers from the somatic disciplines should consider psychiatry-specific peculiarities like the problematic measuring of the treatment outcome. The article reviews at first briefly the pre- 
sent state of quality management in psychiatry and than presents a line of projects on quality improvement in schizophrenia treatment under the umbrella of the German Competence Network for Schizophrenia.

\section{Key words}

schizophrenia - quality management outpatient care - inpatient care guidelines implementation

\section{Literatur}

1. Arbeitsgemeinschaft der Wissenschaftlichen Medizinischen Fachgesellschaften (AWMF)/Ärztliche Zentralstelle Qualitätssicherung (ÄZQ) (Hrsg.). Das LeitlinienManual. Entwicklung und Implementierung von Leitlinien in der Medizin. ZeaFQ 2001; 95 (Suppl. 1)

2. Cording C, Gaebel W, Sprengler A. Die neue psychiatrische Basisdokumentation. Spektrum Psychiatrie Nervenheilkd 1995; 24: 3-41

3. Deutsche Gesellschaft für Psychiatrie, Psychotherapie und Nervenheilkunde (Hrsg.). Behandlungsleitlinie Schizophrenie. Darmstadt: Steinkopff 1998

4. Frick U, Krischker S, Cording C. Freiwillige Krankenhausvergleiche zur externen Qualitätssicherung in der Psychiatrie. Bonn: Bundesministerium für Gesundheit und Soziale Sicherung 2003

5. Gaebel W, Falkai P. Praxisleitlinien in der Psychiatrie. Nervenarzt 1996; 67: 179-181

6. Gaebel W, Janssen B, Schneider F. Qualitätsoptimierung klinischer Schizophreniebehandlung: Ergebnisse und Empfehlungen zur externen Qualitätssicherung. Darmstadt: Steinkopff 2000

7. Gaebel W, Schneider F, Janssen B. Qualitätsmanagement. In: Gaebel W, MüllerSpahn F (Hrsg.). Diagnostik und Therapie psychischer Störungen. Stuttgart: Kohlhammer 2002: 1257-1273

8. Grimshaw JM, Shirran L, Thomas R et al. Changing provider behaviour: an overview of systematic reviews of interventions. Med Care 2001; 39: II2-1145

9. Grol R. Improving the quality of medical care. Building bridges among professional pride, payer profit, and patient satisfaction. JAMA 2001; 286: 2578-2585

10. Janssen B, Menke R, Pourhassan F, Geßner-Özokyay D, Peters R, Gaebel W. Leitlinienimplementierung auf der Basis eines computergestützten Decision-Support-Systems - ein Beitrag zum Qualitätsmanagement in der ambulanten nervenärztlichen Schizophreniebehandlung (Zur Veröffentlichung eingereicht $01 / 2004$ bei Nervenarzt) 11. Janssen B, Burgmann $C$ et al. Externe Qualitätssicherung der stationären Behandlung schizophrener Patienten: Ergebnisse einer multizentrischen Studie. Nervenarzt 2000; 71: 364-372

12. Janssen B, Weinmann $S$ et al. Leitlinienkonformität und Behandlungsergebnisse in der stationären Schizophreniebehandlung: Ein Klinikvergleich. Nervenarzt; online publiziert 24. Juli 2004

13. Janssen B, Weinmann S, Berger M, Gaebel W: Validation of polypharmacy process measures in inpatient schizophrenia. (Zur Veröffentlichung eingereicht 09/2003 bei Schizophrenia Bulletin)

14. Lützenkirchen A. Stärkung oder Schwächung ärztlicher Autonomie? Die medizinische Profession und das Beispiel der evidenzbasierten Medizin aus soziologischer Sicht. Z ärztl Fortbild Qual Gesundh wes 2004; 98: 423-427

15. Menke R, Janssen B, Gaebel W. „Leitliniengestütztes Qualitätsmanagement in der Nervenarztpraxis“ - ein Beitrag zu Entwicklung und Umsetzung eines DiseaseManagement-Programms in der Versorgung schizophrener Patienten. NeuroTransmitter 2001; 11:36-44

16. Richter D. Qualitätsindikatoren für die psychiatrische Versorgung - Eine Übersicht über Kriterien, Methoden und Probleme. Krankenhauspsychiatrie 2004; 15: 1-10

17. Schmacke N. Versorgungsforschung auf dem Weg zu einer Theorie der „letzten Meile“. Gesundh ökon Qual manag 2004; 9: 167-171

18. Weinmann S, Janssen B, Gaebel W. Switching antipsychotics in inpatient schizophrenia care - predictors and outcome. (Zur Veröffentlichung eingereicht)

\section{Korrespondenzadresse:}

Dr. Birgit Janssen

Rheinische Kliniken Düsseldorf

Bergische Landstraße 2

40629 Düsseldorf

birgit.janssen@lvr.de

\section{Theorie, Klinik und Praxis Traumazentrierte Psychotherapie}

Sachsse Ulrich (Hrsg.). Traumazentrierte Psychotherapie. Theorie, Klinik und Praxis. Stuttgart; Schattauer Verlag 2004. 464 Seiten, 15 Abb., 25 Tab., geb., € 49,95. ISBN 3-7945-1971-X.

$\mathrm{T}$ rotz Vielfrauen/Vielmännerbuch sucht der Herausgeber seine persönliche Sichtweise zum Thema dem Leser nahe zu bringen. Das ist sicher auch gelungen und er bedankt sich bei allen für die freundliche Unterstützung, die er dabei hatte.

Es wird die traumazentrierte Psychotherapie abgehandelt, quasi nach allen Richtungen und man ist als Rezensent erstaunt, wie viele Möglichkeiten es gibt, das Thema zu beleuchten.

Dabei bleibt verständlicherweise offen, ob der Sachverhalt stimmt und richtig ist, da wissenschaftlich fundierte Untersuchungen zu diesem Thema kaum vorliegen.

Das Buch stellt für Interessierte eine Fundgrube dar und ist diesem Leserkreis empfohlen.

\section{Prof. F. Reimer, Weinsberg}

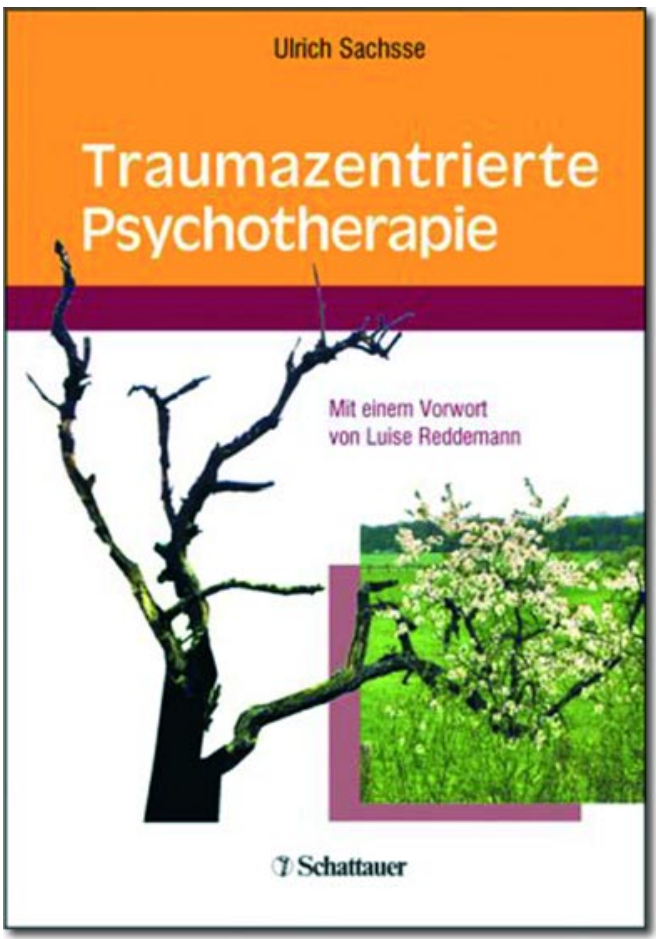

